AN AUTOMATIC DEVICE FOR STOPPING ENGINES.

An improved device has been invented by John J. Kaye, of Newburg, N. Y., which is designed for stopping an engine in an emergency either automatically or manually, without the aid of an engineer. Fig. 1 is a side elevation of an engine with the improvement applied. Fig. 2 is a sectional plan view of a gate-valve eluployed. Fig. 3 is a sectional side elevation of the gate-valve. Fig. 4 shows a speed-limiting arrangement.

Between the steam-supply pipe and the boileragatevalve is arranged, which suddenly shuts off the steam in case of an emergency or when the engine runs at a

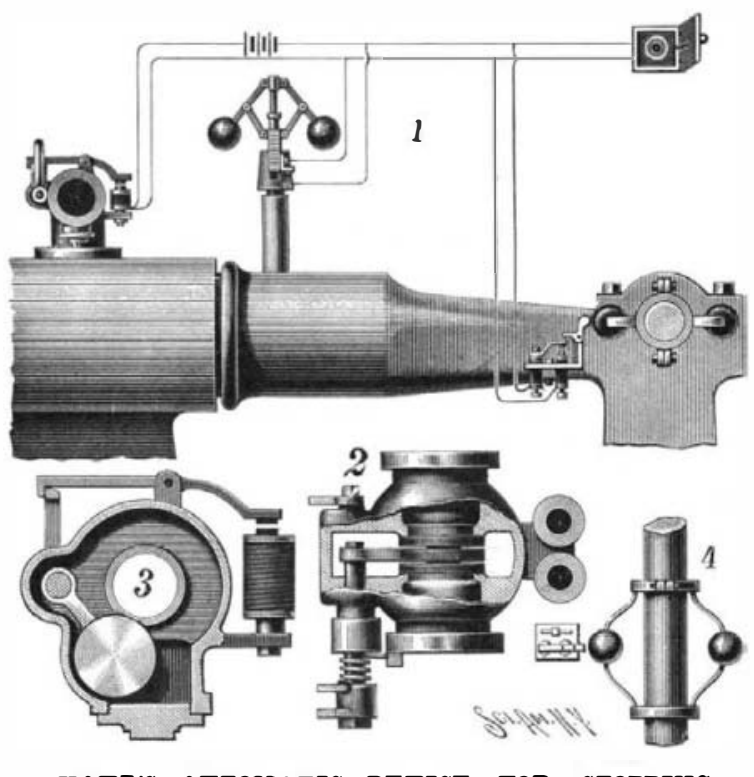

KAYE'S AUTOMATIC DEVICE FOR STOPPING ENGINES.

speed in excess of the normal. The gate consists of disks hung on an arm secured to the inner end of a shaft. A spring, coiled about the shaft, serves to close the valve. The valve is normally held open by means of a locking device consisting of a U-shaped arm connected with the valve shaft, which arn is engaged at its middle by the hook end of the armature lever of a pair of electromagnets. These electromagnets are in a main circuit containing a battery and a pushbutton incased in a box located at any distance from the engine, so that in case of emergency, the button can be pressed to complete the circuit and cause the electromagnets to attract the armature-lever, thereby moving the hook out of engugement with the arm and causing the spring to turn the shaft in order to swing the gate-valve into closed position. The steam is thus cut off almost instantaneously. A number of push buttons can be distributed about a factory, all connected. with the engine furnishing power, so that all machinery can be stopped whenever it may be necessary.

A branch-circuit is connected with a speed-limit device consisting of springs secured at one end to a support fixed to the engine-shaft, and at the other end to port insed to the engine-shaft, and at the other en a call slining on the engine-shaft. Balls are held on he springs (Fig. 4), and are foreed outward when the speed becomes excessive, thereby impinging against a lever which automatically. operates a circuit-closer and actuates the gate-valve in the manner described.

A second branch-circuit is connected with a circuit closer applied to the governor. When for any cause the governor refuses to operate and the balls hang down, then the circuit is automatically closed and the steam cut off by the gate-valve. Hence when the governor and co-operating parts are injured, the engine is almost instantly stopped. The gate-valve when it has been closed can be reset by means of a handle attached to the valve-shaft.

\section{NODES AND LOOPS}

BY M. J. HOFERER,

To anyone who has ever attempted to explain the action of sound waves in an organ pipe, the contrivance shown will at once commend itself. Ordinary textbook diagrans serve only to bewilder the student on this somewhat intricate point in physics. He is constrained to flx his attention at phe tudinal waves meeting each other at every possible phase and always under the guise of sine curves. What a relief could he turn from the lifeless page of his book to an illurninated screen where the direct and reflected waves might be seen moving toward each other with perfect distinctness, and the resultant showing itself at every instant with infallible accuracy, and where, above all, the corresponding longitudinal waves might be seen advancing side by side with their disguised representatives! Such precisely is the result obtained by the sound-wave lantern-slide. It was devised by the writer under pressure of the above diffeulty.

It consists of a wooden frame of a size suited to the lantern and four half-inch rollers about which moves a transparent belt of celluloid film. Upon this is traced a sine curve together with the corresponding longitudinal waves represented by dots properly spaced. The two inner rollers serve merely to bring both parts of the belt together into focus, as shown in the separate view. A wire sine curve which revolves once for every wave on the belt represents the resultant of the direct and reflected wavesat every possible phase of combination. This wire sine curve is connected with one of the rollers by means of small cogwheels. Now, by turning the thumb-screw at the end of the slide, the rollers are made to revolve, and one part of the belt to move to the right and the other to the left, thereby causing the waves to advance toward one another continuously. The wire sine curve keeps exact pace with the two waves and shows at every instant the algebraic sum of their combined ordinates. The perpendicular dark lines crossing the field mark the position of the stationary nodes and loops.

The practical results of the apparatus will be better understood by inspecting the accompanying diagrams. The first five show the direct and reflected waves under various relations of phase together with their resultants, which are represented by the heavy sine curves. In No. 1, where the waves are exactly opposed to one another, the resultant is zero, and this is represented on the screen by the wire when its curves are in the same plane as the eye of the observer. The other four diagrams show a gradual increase in the resultant, until in No. 5 it is almost a maximum.

Nos. 6 and 7 are a representation of two ways in which the curves may be traced upon the film. In No. 6 the ordinates of the sine curve represent displacement of particles: the ordinates above $o \approx$ displace-

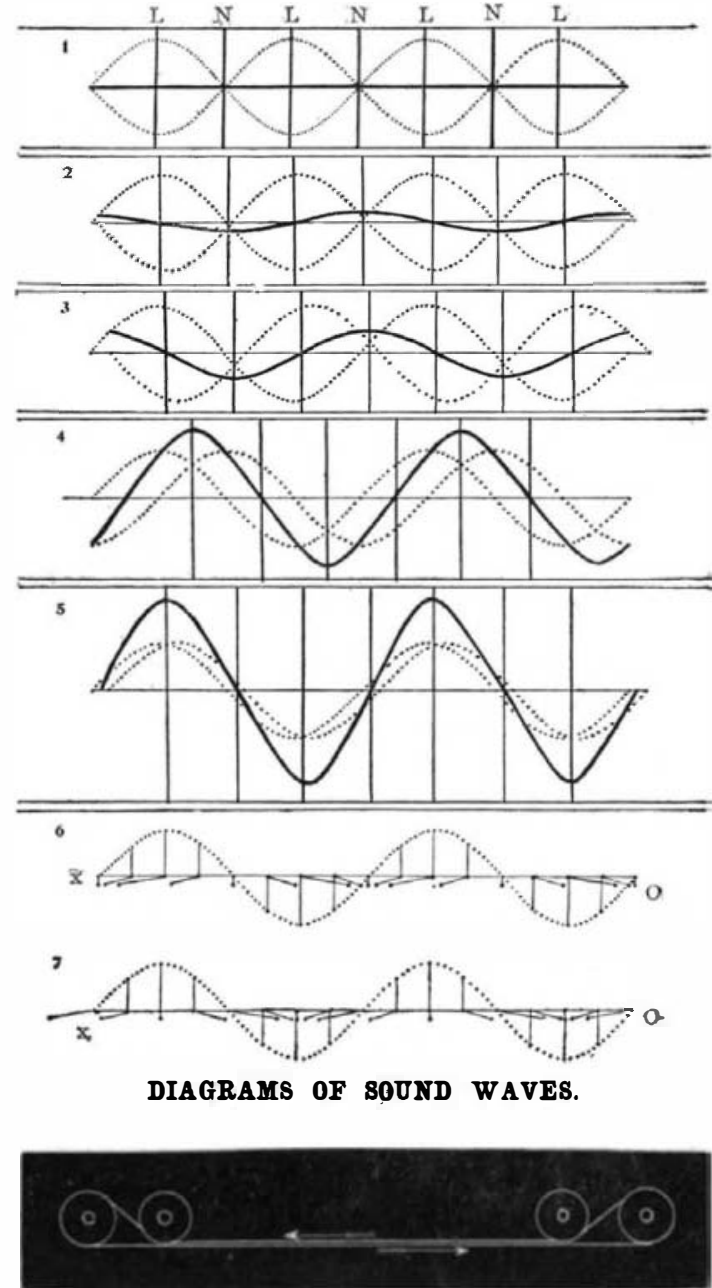

EDGE VIEW OF THE ENDLESS FILM. ment to the right of the point of rest, and those below $o x$ displacement to the left. In this case, however, an allowance of half a wave length must be made in the position of the resultant, owing to the fact that right and left have interchanged places in the reflected wave. This point is beautifully shown upon the screen. In No. 7 the ordinates represent different degrees of rarefaction and condensation, which, not bearing the rela tions of right and left, are not disturbed by being reversed in the reflected wave. The displacement method, however, has the advantage of being more

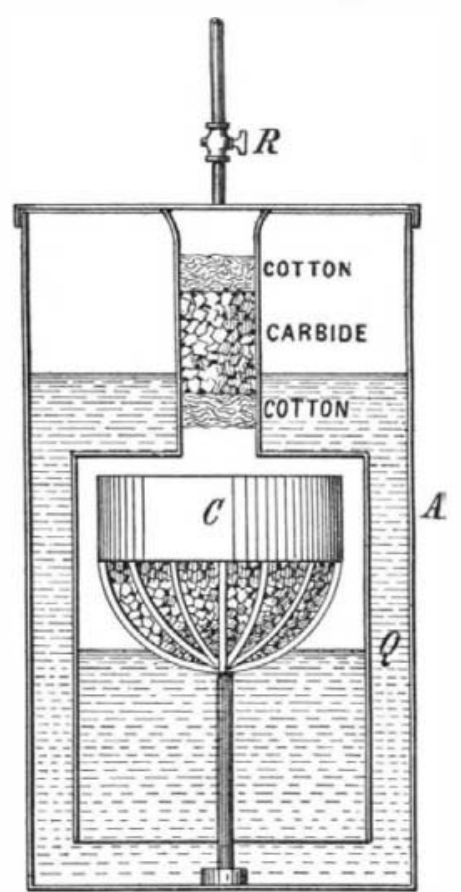

SERPOLLET ACETYLENE GENERATOR.

realistic and definite, as in this case the transverse displacement in the sine curve corresponds exactly to the longitudinal displacement of air particles in sound waves.

\section{BERPOLLET ACETYLENE GENERATOR}

A new form of acetylene generator has been invented in France by Messrs. Letang and Serpollet, in which a constant and regular production is aimed at, the eupply ceasing when the gas is not used. The inventors have obtained good results with this apparatus in the lighting of cars and automobiles, and the Compagnie Génerale des Omnibus have lately made trials of this system with reference to lighting the cars of the Louvre-Vincennes line. The carbide of calcium is treated by immersing it in petroleum for several weeks until it is well impregnated, and afterward giving it an exterior coating of glucose. The presence of the latter gives rise to the formation of a soluble organic salt of calcium, which facilitates the reaction and the proper disengagement of gas. The petroleum seems to protect the carbide from the action of the air. To this product the inventors have given the name of "acetylithe."

The generator consists of an outer cylinder, $A$, of tinned brass, filled with water to three-fourths of its height. To the bottom is fixed a basket-like receptacle, $C$, containing the carbide. The cylinder, $Q$, open at the bottom, is attached to the cover, and when lowered into the water causes the level to descend to a point below the recipient, $C$. If however, the cock, $R$, is opened, this level rises, and the water reaches the carbide, causing an evolution of gas. Upon closing the cock, the pressure of the gas causes the water-level to redescend, and the production ceases. The upper part of the cylinder, $Q$, is narrowed, and contains a layer of untreated carbide between two layers apparatus, with umption of 3 kilogrammes of acetylithe, will generate one cubic meter of acetylene, which burns from 7 to 8 hours in the group of burners devised by the inventors, giving 200 candle power. In the cars of the Compagnie Generale, the tests have been made with five lamps with a total of 50 candle power, consuming 270 grammes of acetylithe per hour Owing to the favorable results obtained in these favorable results obtained will be used on several of the lines now in construction in view of the Exposition.

Among the photographic questions to be dis cussed at the meeting of the Congrès des Socieres Savantes, which will be held at the Sorbonne this year, are the following: Orthochromatic photography ; researches relating to photographic shutters; preparation of photographic surfaces having the fineness of collodion and albumen. and which may be employed in the same manner as the plates now in use; researches in development of plates, printing of different papers, toning and other operations. This meeting promises to be an important one, and will doubtless bring out a number of interesting researches. 